\title{
revengc: An $R$ package to Reverse Engineer Summarized Data
}

by Samantha Duchscherer, Robert Stewart, and Marie Urban

\begin{abstract}
Decoupled (e.g. separate averages) and censored (e.g. $>100$ species) variables are continually reported by many well-established organizations, such as the World Health Organization (WHO), Centers for Disease Control and Prevention (CDC), and World Bank. The challenge therefore is to infer what the original data could have been given summarized information. We present an $R$ package that reverse engineers censored and/or decoupled data with two main functions. The cnbinom. pars() function estimates the average and dispersion parameter of a censored univariate frequency table. The $\mathrm{rec}($ ) function reverse engineers summarized data into an uncensored bivariate table of probabilities.
\end{abstract}

\section{Introduction}

The revengc $\mathrm{R}$ package was originally developed to help model building occupancy (Stewart et al., 2016). Household size and area of residential structures are typically found in any given national census. If a census revealed the raw data or provided a full uncensored contingency table (household size $\times$ area), computing interior density as people per area would be straightforward. However, household size and area are often reported as decoupled variables (separate univariate frequency tables, average values, or a combination of the two). Furthermore, if a contingency table is provided, it typically left $(<, \leq)$, right $(>, \geq,+)$, and interval $(-)$ censored. This summarized information is problematic for numerous reasons. How can a people per area ratio be calculated when no affiliation between the variables exist? If a census reports a household size average of 5.3, then how many houses are there with 1 person, 2 people, .., 10 people? If a census reports that there are 100 houses in an area of $26-50$ square meters, then how many houses are in $26,27, \ldots, 50$ square meters?

A tool that approximates negative binomial parameters from a censored univariate frequency table as well as estimates interior cells of a contingency table governed by negative binomial and/or Poisson marginals can also be useful for other areas ranging from demographic and epidemiological data to ecological inference problems. For example, population and community ecologists could unpack censored organism counts or average life expectancy values. Moreover, other summarized examples include the average number of births, the number of new disease cases, the number of mutations in a gene or average mutation rate, etc. We attempt to accommodate for various application of count data by offering five scenarios that can be reverse engineered:

1. cnbinom. pars () - An univariate frequency table estimates an average and dispersion parameter

2. $\operatorname{rec}()$ - Decoupled averages estimates an uncensored contingency table of probabilities

3. $\operatorname{rec}()$ - Decoupled frequency tables estimates an uncensored contingency table of probabilities

4. $\operatorname{rec}()$ - An average and frequency table estimates an uncensored contingency table of probabilities

5. $\operatorname{rec}()$ - A censored contingency table estimates an uncensored contingency table of probabilities

This paper proceeds with our reverse engineering methodology for the two main functions, cnbinom. pars() and $\operatorname{rec}()$. We provide an in-depth analysis of how we implemented both the negative binomial and Poisson distribution as well as the truncdist (Nadarajah and Kotz, 2006; Novomestky and Nadarajah, 2016) and mipfp R package (Barthélemy and Suesse, 2018a,b). Since the revengc package has specific input requirements for both cnbinom. $\operatorname{pars}()$ and $\operatorname{rec}()$, we continue with an explanation of how to format the input tables properly. We then provide coded examples that implements revengc on national census data (household size and area) and end with concluding remarks.

\section{Methodology: cnbinom. pars()}

The methodology for the cnbinom. pars() function is relatively straightforward. To estimate an average $\mu$ and dispersion $r$ parameter, a censored frequency table is fit to a negative binomial distribution using a maximum log-likelihood function customized to handle left $(<, \leq)$, right $(>, \geq,+)$, and interval $(-)$ censored data. To show an example, first recall the negative binomial distribution $P(X=x \mid \mu, r)$ parameterized as a distribution of the number of failures $X$ before the $r^{\text {th }}$ success in independent trials 
(1). With success probability $p$ in each trail, $r \geq 0$ and $0 \leq p \leq 1$ (Lindén and Mäntyniemi, 2011).

$$
\begin{aligned}
P(X=x \mid r, p)=\left(\begin{array}{c}
x+r-1 \\
x
\end{array}\right) p^{r}(1-p)^{y} & \equiv P(X=x \mid r, \mu)\left(\begin{array}{c}
x+r-1 \\
x
\end{array}\right)\left(\frac{r}{\mu+r}\right)^{r}\left(\frac{\mu}{\mu+r}\right)^{y} \\
E(X) & =\frac{r(1-p)}{p}=\mu \\
V(X) & =\frac{r(1-p)}{p^{2}}=\mu+\frac{\mu^{2}}{r}
\end{aligned}
$$

Now consider an arbitrary censored frequency table $x$ that has a combination of left censored $(x<c)$, interval censored $(a \leq x \leq b)$, and right censored $(x>d)$ data (i.e. $a, b, c$, and $d$ represent the censoring limits). The optimal $\mu$ and $r$ parameter for $x$ maximizes its custom log-likelihood function (2).

$$
\begin{aligned}
L(\mu, r \mid x)_{\log }= & \\
& +\sum_{x<c} \log (P(x<c \mid \mu, r)) \\
& +\sum_{a \leq x \leq b} \log (P(a \leq x \leq b \mid \mu, r)) \\
& +\sum_{x>d} \log (P(x>d \mid \mu, r))
\end{aligned}
$$

\section{Methodology: $\operatorname{rec}()$}

\section{Overview}

$\mathrm{rec}()$ is a statistical approach that estimates the probabilities of a 'true' contingency table given summarized information: two averages, two univariate frequency tables, a combination of an average and univariate frequency table, and a censored contingency table. Figure 1 presents a methodology workflow.

\section{Negative Binomial and Poisson Distribution}

When only an average is provided, we assume the average and variance are equal and rely on a Poisson distribution (i.e. the probability of observing $x$ events in a given interval is given by Equation 3). We understand that there are many cases where data has more variation than what is indicated by the Poisson distribution (e.g. overdispersion). However, with limited data, the Poisson distribution is implemented due to its convenient property of having only one parameter, $\lambda=$ average. For the cases with more data (e.g. univariate frequency table(s) or censored contingency table), we account for dispersion by relying on the more flexible negative binomial distribution (1). Hence, in these cases, the cnbinom.pars() function estimates the optimal average $\mu$ and dispersion $r$ parameters.

$$
\begin{aligned}
P(X=x) & =e^{-\lambda} \frac{\lambda^{x}}{x !} \\
E(X) & =\lambda \\
V(X) & =\lambda
\end{aligned}
$$

\section{The truncdist $R$ package}

With the negative binomial ( $\mu$ and $r$ ) and/or Poisson $(\lambda)$ parameters, $\operatorname{rec}()$ calculates truncated distributions to represent uncensored row (Xlowerbound: Xupperbound) and column (Ylowerbound:Yupperbound) margins. Calculations use the truncdist $\mathrm{R}$ package, and to provide a reference, Equation 4 gives the probability density function of a truncated $X$ distribution over the interval $(a, b]$ (i.e. the negative binomial and/or Poisson probability density function is represented by $g(\cdot)$ and their corresponding cumulative distribution function is denoted by $G(\cdot)$ ). Note, truncated distributions are very practical in this context because the distributions (margins) are restricted to a desired row and column length.

$$
f_{X}(x)= \begin{cases}\frac{g(x)}{G(b)-G(a)}, & \text { if } a<x \leq b \\ 0, & \text { otherwise }\end{cases}
$$

The $(a, b]$ interval needed for both $X$ (row of contingency table) and $Y$ (column of contingency table) can be selected intuitively or with a brute force method. If $\operatorname{rec}()$ outputs a final contingency 


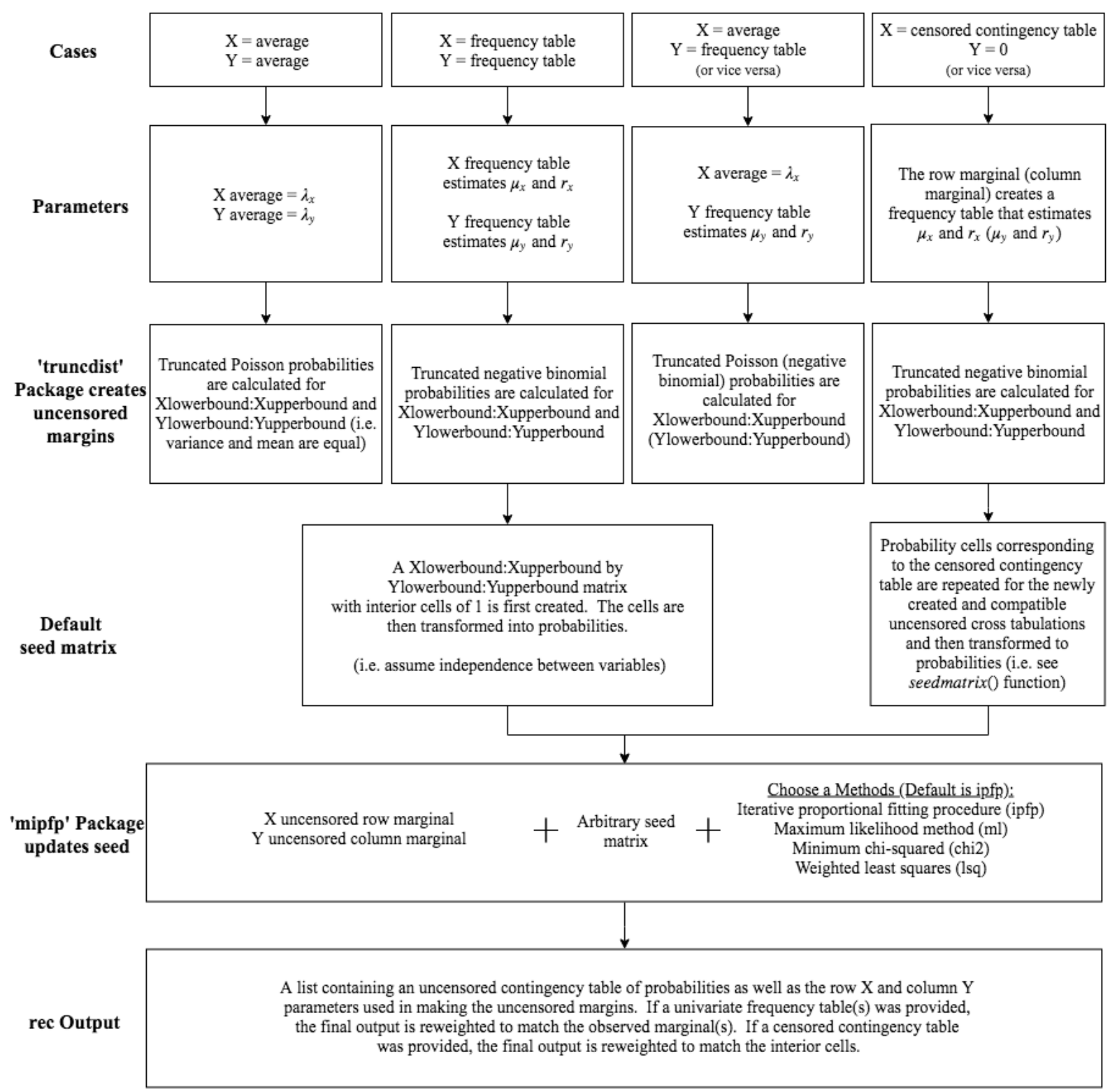

Figure 1: Workflow of rec() function. 
table with higher probabilities near the edge(s) of the table, then it would make sense to increase the range of the bound(s). For both variables, this would just involve making the lower bound less, making the upper bound more, or doing a combination of the two. The opposite holds true as well. If the final contingency table in $\operatorname{rec}()$ has very low probabilities near the edge(s) of the table, the range of the particular bound(s) should be decreased.

\section{The mipfp R package}

rec() utilizing the mipfp $\mathrm{R}$ package to calculate cross tabulation probability estimates, and mipfp requires fixed marginals, a seed estimation method, and a seed matrix. The row and column marginals are uncensored truncated distributions (see The truncdist $R$ package section) while opportunities for sensitivity analysis are presented with the seed estimation method and seed matrix. For example, mipfp offers four seed estimation methods (Table 1); the default method in $\operatorname{rec}()$ is the iterative proportional fitting procedure. Although the algorithms vary, they all adjust cell proportions $p_{x y}$ in a $X \times Y$ contingency table to known marginal probabilities, $\pi_{x+}$ and $\pi_{+y}$ (i.e. all interior cell estimates $\widehat{\pi}_{x y}$ are subject to marginal constraints (5)). For an expanded explanation of these methods, please refer to Little and Wu (1991) and Suesse et al. (2017).

$$
\begin{array}{ll}
\sum_{y} \hat{\pi}_{x+} & (x=1, \ldots, X) \\
\sum_{x} \hat{\pi}_{+y} & (y=1, \ldots, Y)
\end{array}
$$

\begin{tabular}{ll}
\hline Method & Calculate $\hat{\pi}_{x y}$ by \\
\hline Iterative proportional fitting procedure - ipfp & Minimizing $\sum_{x} \sum_{y} \hat{\pi}_{x y} \ln \left(\hat{\pi}_{x y} / p_{x y}\right)$ \\
Maximum likelihood method - ml & Maximizing $\sum_{x} \sum_{y} p_{x y} \ln \left(\hat{\pi}_{x y}\right)$ \\
Minimum chi-squared - chi2 & Minimizing $\sum_{x} \sum_{j}\left(\hat{\pi}_{x y}-p_{x y}\right)^{2} / \hat{\pi}_{x y}$ \\
Weighted least squares - lsq & Minimizing $\sum_{x} \sum_{y}\left(p_{x y}-\hat{\pi}_{x y}\right)^{2} / p_{x y}$ \\
\hline
\end{tabular}

Table 1: Algorithms to generate estimated cross tabulations.

The seed matrix input can be arbitrary, but $\operatorname{rec}()$ provides reasonable defaults. For the decoupled cases (two averages, two tables, or a combination of a table and average), the absence of additional information makes it difficult to say much about the joint distribution. Therefore, $\operatorname{rec}()$ assumes independence between the variables, which is equivalent in making the $X \times Y$ seed a matrix of ones; $\operatorname{rec}()$ converts the matrix of ones to probabilities. When a censored contingency table is provided, independence does not have to be assumed and the interior cells can be weighted. $\operatorname{rec}($ ) creates the default seed matrix by first repeating probability cells, which correspond to the censored contingency table, for the newly created and compatible uncensored cross tabulations. Just as in the decoupled cases, the cell values in this matrix are also changed to probabilities. To see an example of the default seed for a censored contingency table, see Worked examples section.

\section{Usage}

\section{cnbinom.pars()}

The cnbinom.pars() function has the following format:

cnbinom.pars (censoredtable)

where censoredtable is a frequency table (censored and/or uncensored). A data.frame and matrix are acceptable classes. See Data entry section for formatting. The output is a list consisting of an estimated average $\mu$ and dispersion parameter $r$.

rec()

The $\operatorname{rec}($ ) function has the following format:

$\operatorname{rec}(X, Y, X l o w e r b o u n d$, Xupperbound, Ylowerbound, Yupperbound, seed.matrix, seed.estimation.method) 
where

- X: Argument can be an average, a univariate frequency table, or a censored contingency table. The average value should be a numeric class while a data.frame or matrix are acceptable table classes. Y defaults to NULL if $X$ argument is a censored contingency table. See Data entry section for formatting.

- $Y$ : Same description as $X$ but this argument is for the $Y$ variable. $X$ defaults to NULL if $Y$ argument is a censored contingency table.

- Xlowerbound: A numeric class value to represent the left bound for X (row in contingency table). The value must strictly be a non-negative integer and cannot be greater than the lowest category/average value provided for $X$ (e.g. the lower bound cannot be 6 if a table has ' $<5^{\prime}$ as a $X$ or row category).

- Xupperbound: A numeric class value to represent the right bound for $X$ (row in contingency table). The value must strictly be a non-negative integer and cannot be less than the highest category/average value provided for X (e.g. the upper bound cannot be 90 if a table has ' $>100$ ' as a $\mathrm{X}$ or row category).

- Ylowerbound: Same description as Xlowerbound but this argument is for Y (column in contingency table).

- Yupperbound: Same description as Xupperbound but this argument is for Y (column in contingency table).

- seed.matrix: An initial probability matrix to be updated. If decoupled variables is provided the default is a Xlowerbound:Xupperbound by Ylowerbound:Yupperbound matrix with interior cells of 1 , which are then converted to probabilities. If a censored contingency table is provided the default is the seedmatrix()\$Probabilities output.

- seed.estimation. method: A character string indicating which method is used for updating the seed.matrix. The choices are: "ipfp", "ml", "chi2", or "lsq". Default is "ipfp".

The output is a list containing an uncensored contingency table of probabilities (rows range from Xlowerbound:Xupperbound and the columns range from Ylowerbound:Yupperbound) as well as the row and column parameters used in making the margins for the mipfp $\mathrm{R}$ package.

\section{Data entry}

The input tables are formatted to accommodate most open source data. The univariate frequency table used in cnbinom. pars() and/or $\operatorname{rec}()$ needs to be a data.frame or matrix class with two columns and $n$ rows. The categories must be in the first column with the frequencies or probabilities in the second column. Row names should never be placed in this table (the default row names should always be 1:n). Column names can be any character string. The only symbols accepted for censored data are listed below.

- Left censored symbols: $<, \leq, \mathrm{L}$, and LE

- Interval censored symbols: - and I (symbol has to be placed in the middle of the two category values)

- Right censored symbols: $>, \geq,+, \mathrm{G}$, and GE

- Uncensored symbol: no symbol (only provide category value)

Note, less than or equal to $(\leq$ and LE) is not equivalent to less than $(<$ and $\mathrm{L})$ and greater than or equal to $(\geq,+$, and GE) is not equivalent to greater than ( $>$ and G). revengc also uses closed intervals. Table 2 shows three different examples that all give the same cnbinom. pars() output.

The censored contingency table for $\mathrm{rec}()$ has a similar format. The censored symbols should follow the requirements listed above. The table's class can be a data.frame or a matrix. The column names should be the $Y$ category values. The first column should be the $X$ category values and the row names can be arbitrary. The inside of the table are $X \times Y$ frequencies or probabilities; the tabulations must be non-negative if the seed.estimation.method = "ipfp" or strictly positive if the seed.estimation.method is "ml", "lsq", or "chi2". The row $X$ and column $Y$ marginal totals need to be placed in this table. The top left, top right, and bottom left corners of the table should be NA or blank. The bottom right corner can be a total cross tabulation sum value, NA, or blank. Table 3 is a formatted example. 


\begin{tabular}{|c|c|c|c|c|c|}
\hline Category & Frequency & Category & Frequency & Category & Frequency \\
\hline$\leq 6$ & 11800 & LE 6 & 11800 & $<7$ & 11800 \\
\hline $7-12$ & 57100 & 7 I 12 & 57100 & 7 I 12 & 57100 \\
\hline $13-19$ & 14800 & 13 I 19 & 14800 & $13-19$ & 14800 \\
\hline $20+$ & 3900 & GE 20 & 3900 & $\geq 20$ & 3900 \\
\hline
\end{tabular}

Table 2: Examples of correctly formatted univariate tables.

\begin{tabular}{ccccc}
\hline NA & $<20$ & $20-30$ & $>30$ & NA \\
$<5$ & 18 & 19 & 8 & 45 \\
$5-9$ & 13 & 8 & 12 & 33 \\
$\geq 10$ & 7 & 5 & 10 & 21 \\
NA & 38 & 32 & 31 & NA \\
\hline
\end{tabular}

Table 3: Example of a correctly formatted bivariate table.

\section{Formatting tables in $\mathbf{R}$}

The code below shows how to format these tables properly in R.

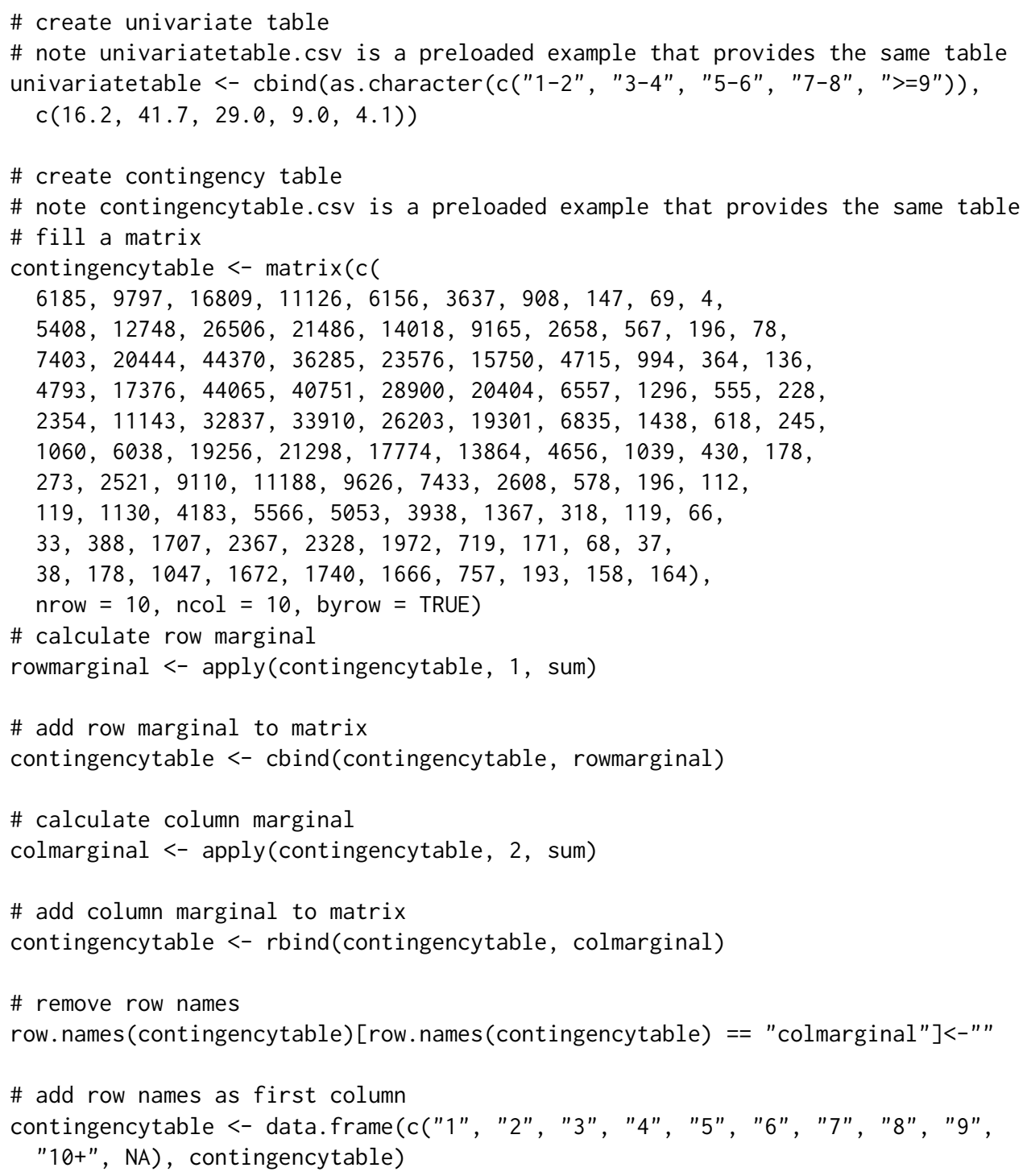


\# add column names

colnames (contingencytable) <- c(NA, "<20", "20-29", "30-39", "40-49",

"50-69", "70-99", "100-149", "150-199", "200-299", "300+", NA)

\section{Worked examples}

\section{Nepal}

A Nepal Living Standards Survey (Government of Nepal, National Planning Commission Secretariat, 2011) provides both a censored table and average for urban household size. We use the censored table to show that the cnbinom. pars() function calculates a close approximation to the provided average household size (4.4 people). Note, there is overdispersion in the data.

\# revengc has the Nepal household table preloaded as univariatetable.csv cnbinom. pars (censoredtable $=$ univariatetable.csv)

\section{Indonesia}

In 2010, the Population Census Data - Statistics Indonesia provided over 60 censored contingency tables containing household member size by floor area of dwelling unit (square meter) (Statistics Indonesia, 2010). The tables are separated by province, urban, and rural. Here we use the rural Aceh Province table to show the multiple coding steps and functions implemented inside rec(). This allows the user to see a methodology workflow in code form. The final uncensored household size by area estimated probability table, which implemented the "ipfp" seed estimation method and default seed matrix, has rows ranging from 1 (Xlowerbound) to 15 (Xupperbound) people and columns ranging from 10 (Ylowerbound) to 310 (Yupperbound) square meters.

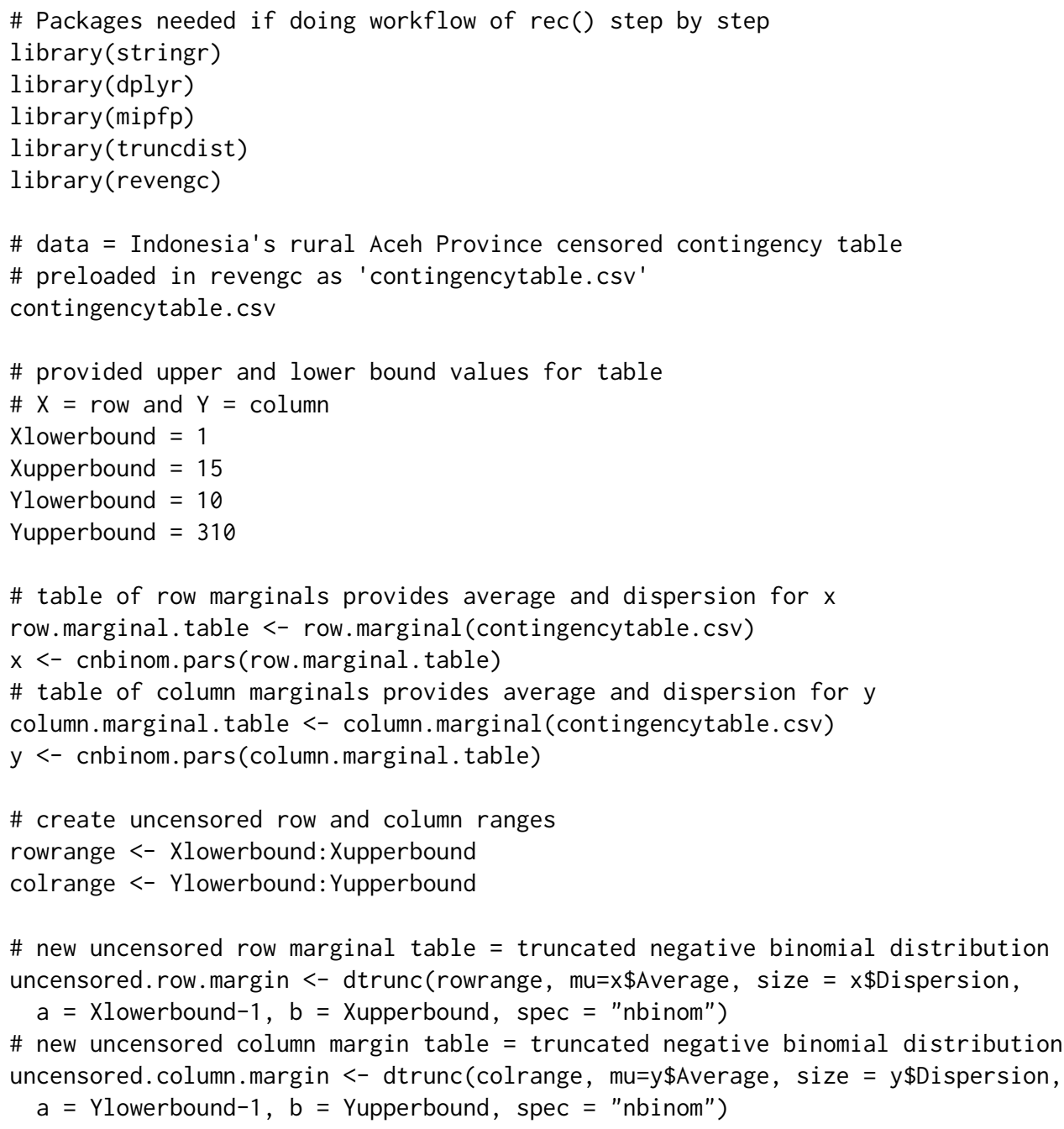




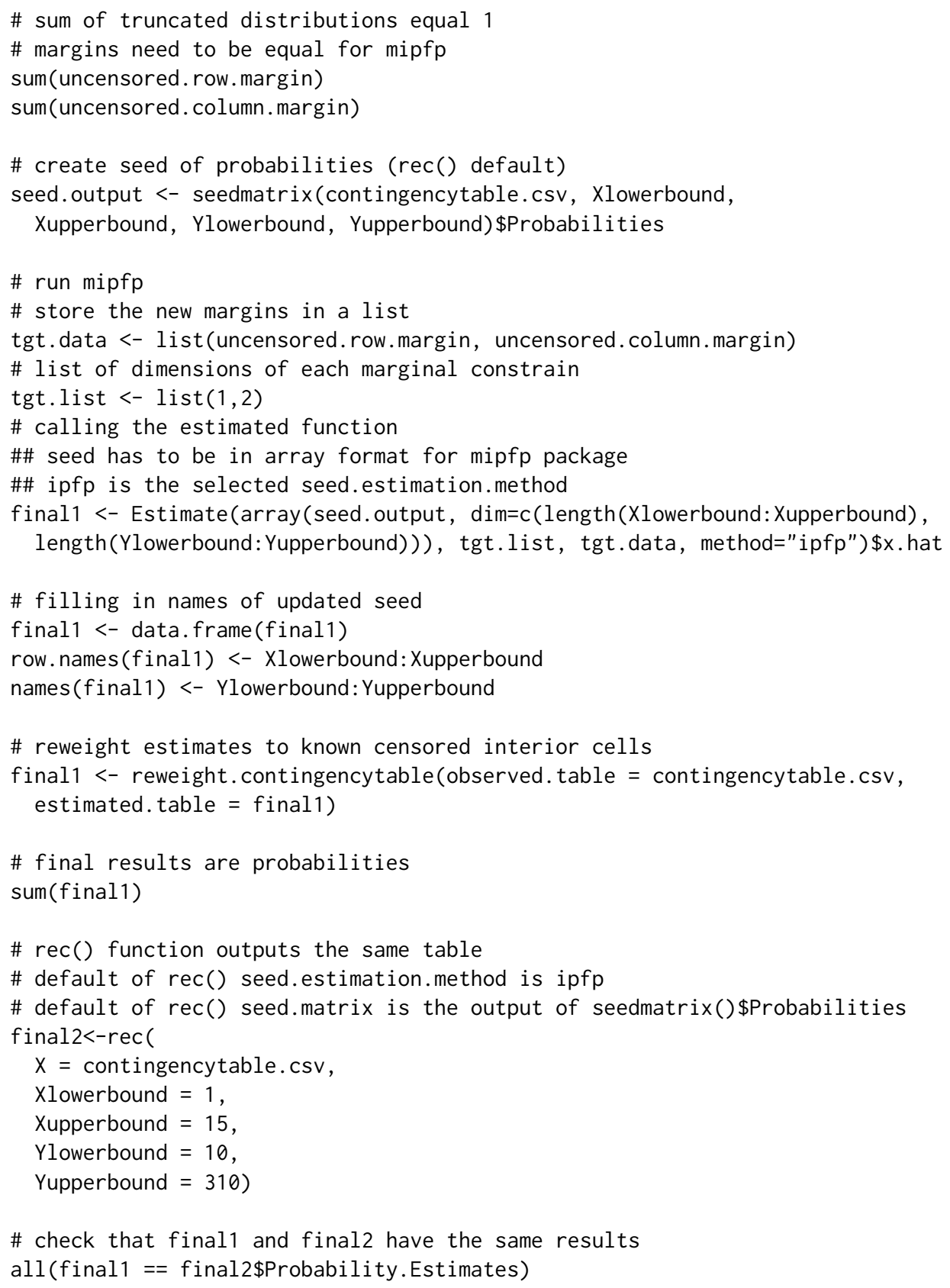

\section{Conclusion}

revengc was designed to reverse engineer summarized and decoupled variables with two main functions: cnbinom. pars() and $\operatorname{rec}()$. Relying on a negative binomial distribution, cnbinom. pars() approximates the average and dispersion parameter of a censored univariate frequency table. $\operatorname{rec}()$ fills in missing interior cell values from observed aggregated data (e.g. decoupled average(s) and/or censored frequency table(s) or a censored contingency table). It is worth noting the required assumptions in $\operatorname{rec}()$. For instance, $\operatorname{rec}()$ relies on a Poisson distribution when only an average is provided, which is assuming the variance and average are equal. More descriptive input variables, such as univariate frequency tables or contingency tables, can account for dispersion found in data. However, independence between decoupled variables still has to be assumed when there is no external information about the joint distribution. For these reasons, revengc provides two options for sensitivity analysis: the seed matrix and the method used in updating the seed matrix are both arbitrary inputs. 


\section{Acknowledgments}

This manuscript has been authored by employees of UT-Battelle, LLC, under contract DE-AC0500OR22725 with the US Department of Energy. Accordingly, the United States Government retains a nonexclusive, paid-up, irrevocable, worldwide license to publish or reproduce the published form of this manuscript, or allow others to do so, for United States Government purposes. The publisher, by accepting the article for publication, acknowledges the above-mentioned conditions.

\section{Bibliography}

J. Barthélemy and T. Suesse. mipfp: An R package for multidimensional array fitting and simulating multivariate bernoulli distributions. Journal of Statistical Software, Code Snippets, 86(2):1-20, 2018a. URL https://doi.org/10.18637/jss.v086.c02. [p114]

J. Barthélemy and T. Suesse. mipfp: Multidimensional Iterative Proportional Fitting and Alternative Models, 2018b. URL https: //CRAN. R-project. org/package=mipfp. R package version 3.2.1. [p114]

Government of Nepal, National Planning Commission Secretariat. Nepal living standards survey. Technical report, Central Bureau of Statistics, 2011. [p120]

A. Lindén and S. Mäntyniemi. Using the negative binomial distribution to model overdispersion in ecological count data. Ecology, 92(7):1414-1421, 2011. URL https://doi . org/10.1890/10-1831.1. [p115]

R. J. Little and M.-M. Wu. Models for contingency tables with known margins when target and sampled populations differ. Journal of the American Statistical Association, 86(413):87-95, 1991. URL https://doi.org/10.2307/2289718. [p117]

S. Nadarajah and S. Kotz. R programs for truncated distributions. Journal of Statistical Software, Code Snippets, 16(2):1-8, 2006. URL https://doi.org/10.18637/jss.v016.c02. [p114]

F. Novomestky and S. Nadarajah. truncdist: Truncated Random Variables, 2016. URL https: //CRAN. Rproject. org/package=truncdist. R package version 1.0-2. [p114]

Statistics Indonesia. Household by floor area of dwelling unit and households member size. Technical report, The 2010 Indonesia Population Census, 2010. [p120]

R. Stewart, M. Urban, S. Duchscherer, J. Kaufman, A. Morton, G. Thakur, J. Piburn, and J. Moehl. A Bayesian machine learning model for estimating building occupancy from open source data. Natural Hazards, 81(3):1929-1956, 2016. URL https://doi .org/10.1007/s11069-016-2164-9. [p114]

T. Suesse, M.-R. Namazi-Rad, P. Mokhtarian, and J. Barthélemy. Estimating cross-classified population counts of multidimensional tables: an application to regional Australia to obtain pseudo-census counts. Journal of Official Statistics, 33(4), 2017. URL https://doi.org/10.1515/jos-2017-0048. [p117] 
Samantha Duchscherer

Oak Ridge National Laboratory

1 Bethel Valley Road Oak Ridge, TN 37831

USA

ORCiD: 0000-0002-2023-3106

sam. duchscherer@gmail.com

Robert Stewart

Oak Ridge National Laboratory

1 Bethel Valley Road Oak Ridge, TN 37831

USA

ORCiD: 0000-0002-8186-7559

stewartrn@ornl.gov

Marie Urban

Oak Ridge National Laboratory

1 Bethel Valley Road Oak Ridge, TN 37831

USA

ORCiD: 0000-0001-9571-832X

urbanml@ornl.gov 